\title{
Ga-68-FAPI as diagnostic tool in sarcoma: Data from the FAPI-PET prospective observational trial
}

Running title: FAPI-PET in sarcoma

Lukas Kessler ${ }^{1,2}$, Justin Ferdinandus ${ }^{1,2}$, Nader Hirmas ${ }^{1,2}$, Sebastian Bauer ${ }^{3,2}$, Uta Dirksen ${ }^{4,2}$, Fadi Zarrad $^{1,2}$, Michael Nader ${ }^{1,2}$, Michal Chodyla ${ }^{5,2}$, Aleksandar Milosevic ${ }^{5,2}$, Lale Umutlu ${ }^{5,2}$, Martin Schuler $^{3 ; 2}$, Lars Erik Podleska ${ }^{6,2}$, Hans-Ulrich Schildhaus ${ }^{7,2}$, Wolfgang P. Fendler ${ }^{1,2}$, Rainer Hamacher $^{3,2}$

${ }^{1}$ Department of Nuclear Medicine, University Hospital Essen, University of Duisburg-Essen, Essen, Germany

${ }^{2}$ German Cancer Consortium (DKTK), Partner Site University Hospital Essen, and German Cancer Research Center (DKFZ), Essen, Germany.

${ }^{3}$ Department of Medical Oncology, West-German Cancer Center, University of Duisburg-Essen, Essen, Germany

${ }^{4}$ Department of Pediatrics III, West-German Cancer Center, University Hospital Essen, Essen, Germany.

${ }^{5}$ Department of Diagnostic and Interventional Radiology and Neuroradiology, University Hospital Essen, Essen, Germany

${ }^{6}$ Department of Tumor Orthopedics and Sarcoma Surgery, University Hospital Essen, Essen, Germany.

${ }^{6}$ Department of Pathology, University Hospital Essen, Essen, Germany

\section{Corresponding author:}

Wolfgang Peter Fendler, M.D.

Address: Hufelandstraße 55, 45147 Essen, Germany

Telephone number: +492017232032

Fax number: +49 2017235658

E-mail: wolfgang.fendler@uk-essen.de 
First author:

Lukas Kessler, M.D.

Address: Hufelandstraße 55, 45147 Essen, Germany

Telephone number: +49 2017232032

Fax number: +49 2017235658

E-mail: lukas.kessler@uk-essen.de

Word count: 4916 (incl. References and Tables + Figures)

\section{Keywords:}

Sarcoma, cancer imaging, FAPI, fibroblast activation protein, PET

\section{Abbrevations:}

\begin{tabular}{|l|l|}
\hline FAP & Fibroblast Activation Protein \\
\hline PET & Positron emission tomography \\
\hline CT & Computed tomography \\
\hline MRI & Magnetic resonance imaging \\
\hline FAPI & Fibroblast activation protein inhibitor \\
\hline FAPI-PET & ${ }^{68}$ Ga-FAPI-PET/CT \\
\hline FDG-PET & ${ }^{18}$ F-Fluordesoxyglucose PET/CT or MRI \\
\hline SUV(mean,max) & Standardized uptake value \\
\hline
\end{tabular}




\section{ABSTRACT}

Introduction: Bone and soft tissue sarcomas express fibroblast activation protein (FAP) on tumor cells and associated fibroblast. Therefore, FAP is a promising therapeutic and diagnostic target. Novel radio-labelled FAP-Inhibitors (e.g. $\left.{ }^{68} \mathrm{Ga}-\mathrm{FAPI} 46\right)$ have shown high tumor uptake in positron emission tomography (PET) in sarcoma patients. Here we report endpoints of the FAPI-PET prospective observational trial.

Methods: Forty-seven patients with bone or soft tissue sarcomas undergoing clinical ${ }^{68} \mathrm{Ga}-\mathrm{FAPI}-$ PET were eligible for enrollment into the FAPI-PET observational trial. Of these patients, 43 patients also underwent ${ }^{18} \mathrm{~F}$-Fluordesoxyglucose PET (FDG). The primary study endpoint was the association of ${ }^{68} \mathrm{Ga}-\mathrm{FAPI}-\mathrm{PET}$ uptake intensity and histopathological FAP-expression analyzed with Spearman's r correlation. Secondary endpoints were detection rate, positive predictive value (PPV), interreader reproducibility, and change in management. Datasets were interpreted by two blinded readers.

Results: Primary endpoint was met and the association between FAPI-PET uptake intensity and histopathological FAP-expression was significant (Spearman's $r=0.43$; $p=0.03$ ). By histopathological validation PPV was $1.00(95 \% \mathrm{Cl}, 0.87-1.00)$ on a per-patient and $0.97(95 \% \mathrm{Cl}$, 0.84-1.00) on a per-region basis. In cases with histopathologic validation, 27 of 28 (96\%) confirmed patients and 32 of 34 (94\%) confirmed regions were PET positive resulting in an SE of $0.96(95 \% \mathrm{Cl}, 0.82-1.00)$ on a per-patient and $0.94(95 \% \mathrm{Cl}, 0.80-0.99)$ on a per-region basis. The detection rate on a per-patient basis in FAPI- and FDG-PET was $76.6 \%$ and $81.4 \%$, respectively. In $8(18.6 \%)$ patients FAPI-PET resulted in an upstaging compared to FDG-PET. FAPI-PET readers showed substantial to almost perfect agreement for the defined regions (Fleiss kappa: primary $\mathrm{K}=0.78$; local nodal $\mathrm{K}=0.54$; distant nodal $\mathrm{K}=0.91$; lung $\mathrm{K}=0.86$; bone $\mathrm{K}=0.69$ and other $k=0.65)$. Clinical management changed in $13(30 \%)$ patients after FAPI-PET.

Conclusion: We confirm an association of tumoral FAPI-PET uptake intensity and histopathological FAP expression in sarcoma patients. Further, using blinded reads and independent histopathological validation we report high PPV and sensitivity of FAPI-PET for sarcoma staging. 


\section{INTRODUCTION}

Sarcomas are a heterogenous group of malignant tumors, which derive from a mesenchymal origin. Reliable imaging methods are utterly important in the disease management of these patients, which mainly focus on computer tomography $(\mathrm{CT})$, magnetic resonance imaging (MRI) or hybrid imaging with additional position emission tomography (PET) using ${ }^{18}$ Fluorine-fluoro-2deoxyglucose $\left({ }^{18} \mathrm{~F}-\mathrm{FDG}\right) .{ }^{18} \mathrm{~F}-\mathrm{FDG}$ PET/CT (hereafter FDG-PET) has gained recognition as an efficient imaging modality for sarcomas and has been linked with detection of local recurrence and metastasis, response assessment and prognosis (1-3). Although the downsides of ${ }^{18} \mathrm{~F}-\mathrm{FDG}$ include the false-positive results due to physiological uptake or inflammatory response or falsenegative results due to elevated serum blood glucose levels. Therefore, alternative targeted imaging probes are of increasing interest. The fibroblast-activation-protein is highly expressed in carcinoma-associated fibroblasts (CAFs) in the stroma of various tumor entities $(4,5)$ and activated fibroblasts in stroma tissue to promote wound healing, but is absent in normal adult tissues (6-8). Carcinoma-associated fibroblasts influence tumor cells by producing mediators and can promote tumor angiogenesis, migration and proliferation $(9,10)$. Based on this, a meta-analysis of 15 studies proved that FAP overexpression in solid tumors is associated with poor outcome and is distinctively overexpressed in tumor tissue compared to normal tissue (8). Compared to other tumor entities sarcomas are rather unique in terms of FAP-expression. Depending on their histogenesis and due to their mesenchymal origin, not just carcinoma-associated fibroblasts but sarcoma tumor cells itself often express FAP as well $(11,12)$. It has been proposed that FAP and DPP4 are consistently expressed in bone and soft tissue tumor cells that are histogenetically related to activated fibroblasts/myofibroblasts, irrespective of their malignancy (11). Therefore, FAP is an interesting specific target for diagnostic and therapeutic probes in bone and soft tissue sarcomas. Only recently novel radiolabeled fibroblast-activation-protein-inhibitors (FAPI) have been introduced for theranostic approaches, which showed promising diagnostic value for multiple tumor entities, including sarcomas $(13,14)$. These novel radiotracers captivate because of their 
low background activity, shorter acquisition delay, no need of dietary measurements and a specific imaging target. Additionally, it is possible to link these tracers with strong $\beta$-emitting radionuclides (e.g. ${ }^{177}$ Lutetium and ${ }^{90}$ Yttrium) and use them for FAP-targeted radioligand therapies (15-17). Nonetheless, data on the imaging potential of these radiotracers are scarce and several clinical trials, as ours, have been started to evaluate these tracers in tumors as well as specifically in sarcoma patients (e.g. NCT04457258).

The aim of this prospective observational study was to investigate the association of histopathological FAP-expression and ${ }^{68} \mathrm{Ga}-\mathrm{FAPI}-\mathrm{PET}$ (hereafter FAPI-PET) tracer uptake intensity in bone and soft tissue sarcoma. We further aim to analyze FAPI-PET sensitivity, specificity, PPV, and impact on management.

\section{METHODS}

\section{Study Design and Patients}

Flow of patients is illustrated in Figure 1 (Fig. 1). This is a subgroup analysis of the ongoing FAPI-PET observational trial. Until November 2020 adult patients with sarcomas, who received a clinical FAPI-PET were offered consent for an observational trial conducted at University Hospital Essen (NCT04571086). Prior to enrollment patients gave written consent to undergo FAPI-PET for clinical indication. Patients were enrolled irrespective of prior conventional imaging or treatment. Inclusion criteria were: FAPI-PET scheduled for staging or restaging of sarcoma as part of clinical routine and age $\geq 18$ years. Clinical indications for FAPI-PET were staging of high risk patients, evaluation localization of tumor lesions prior to biopsy or surgery, equivocal imaging results or evaluation of therapeutic options for instance (Tabl. 1). Exclusion criteria were inability to give consent to study, to tolerate a PET scan and pregnant, lactating, or breastfeeding women. Primary endpoint was the association between FAPI PET uptake intensity and 
histopathologic FAP expression. The primary endpoint is met if PET uptake and tissue FAP expression shows significant correlation by Spearman correlation (ordinal data).

Secondary endpoints were:

(a) Detection rate and positive predictive value (PPV) on a per-patient and per-region-basis of FAPI PET for detection of tumor location, confirmed by histopathology/biopsy.

(b) Sensitivity and specificity of FAPI PET on a per-patient and per-region-basis for detection of tumor location confirmed by histopathology/biopsy.

(c) Impact on management.

(d) Interreader reproducibility.

(e) Change in staging/prognostic groups.

An illustration of the timeline of investigations is illustrated in supplemental material (Supplemental Fig. 1). The study was initiated, planned, conducted, analyzed, and published by the investigators. No financial support was received from commercial entities. All reported investigations were conducted in accordance with the Declaration of Helsinki and with the national regulations. This observational trial was registered on clinicaltrails.gov (NCT04571086) and approved by the local Ethics Committee (permits no. 19-8991-BO and 20-9485-BO). Patients gave written informed consent for inclusion in the observational trial.

\section{Imaging}

Clinical PET scans were performed in craniocaudal direction on a Biograph mMR, Biograph mCT or Biograph mCT VISION (Siemens Healthineers, Germany). All ${ }^{68} \mathrm{Ga}-\mathrm{FAPI} 46-\mathrm{PET}$ scans were performed as PET/CT, whereas ${ }^{18} \mathrm{~F}-\mathrm{FDG}-\mathrm{PET}$ scans were performed either as PET/MRI or $\mathrm{PET} / \mathrm{CT}$, depending on the clinical indication, hereafter referred to as FAPI-PET / FDG-PET. Mean injected activity of ${ }^{68} \mathrm{Ga}-\mathrm{FAPI}$ was $144 \pm 36 \mathrm{MBq}$ and of ${ }^{18} \mathrm{~F}-\mathrm{FDG} 214 \pm 102 \mathrm{MBq}$. FAPI-PET images were acquired approx. ten minutes (mean $13.6 \pm 8.5$ min.) post-injection, and FDG-PET images 
60 minutes (mean $69.2 \pm 16.5$ ) post injection. For PET/CT, a diagnostic CT was obtained with the use of a standard protocol ( 80 to $100 \mathrm{~mA}, 120 \mathrm{kV}$ ) prior to the PET scan. Intravenous iodinated contrast was administered in 45 (96\%) patients. For each scan, number of lesions per region and per patient and the size of the lesion with the highest tracer uptake per region were recorded. Any focal tracer uptake higher than the surrounding background and not associated with physiological

uptake was considered suspicious for malignancy. Standardized uptake values (e.g. SUV $\max$ ) of tumor lesions were measured with a region grow algorithm with a threshold of $40 \%$ of the maximal uptake was performed (Syngo.via software; Siemens Healthcare) for the lesion with the highest tracer uptake in the respective cancer site (primary, local nodal, distant nodal, lung, bone metastasis and other organ- / skin- / soft-tissue metastasis). PET/CT and MRI images were read independently by two experienced, blinded nuclear medicine physicians or radiologists each for the respective modalities (PET/CT: J.F., L.K. or MRI: M.C., A.M., respectively). Readers were unblinded for the localisation of primary tumor site. Divergent findings were discussed and reported in a separate consensus session between readers.

\section{Lesion Validation}

All patients were followed up for histopathologic analysis and, if possible, respective FAPimmunohistochemistry. Lesions were included, if FAPI-PET findings could be directly validated with histopathologically proven lesions. Validation was performed by the unblinded local investigators after reviewing images and reports, following prespecified criteria of the study protocol. In patients with histopathological results, positive FAPI-PET findings were validated as true or false-positive. Region negative on FAPI-PET, but with subsequently confirmed tumor lesion by histopathologic analysis, were considered false-negative results. 


\section{Immunohistochemistry}

Biopsy and surgical specimen were stained with standard haematoxylin and eosin and FAPimmunohistochemistry (IHC). For immunohistochemical staining, the tissue slides were incubated with the anti-FAP antibody after antigen retrieval, followed by secondary antibody incubation.

FAP expression was evaluated by one experienced pathologist (H.U.S) under light microscopy with $40 x$ power in accordance with a system by Henry et al.(18). Assessment of the entire tumor tissue on the slide was performed excluding the invasive front or areas of active tumor growth. Semiquantitative analysis of FAP expression was rated following a previously described scoring system (18-20): $0=$ defined as the complete absence or weak FAP immunostaining in $<1 \%, 1+=$ focal positivity in $1 \%$ to $10 \%, 2+=$ positive FAP immunostaining in $11 \%$ to $50 \%, 3+=\geq$ FAP immunostaining in $>50 \%$. Tumor cells and stromal cells were assessed for semiquantitative analysis. The pathologist was blinded to PET findings.

\section{Survey Design}

Referring physicians were asked to complete and return two questionnaires. The first questionnaire was required to assess the existing treatment plan for the patient without the information from FAPI-PET. The second questionnaire inquired about intended management after receipt of the written clinical report and the FAPI-PET images. After return of the second questionnaire, all other pending imaging findings were disclosed. Implementation of intended management was verified by patient file review or information provided by the referring physician.

\section{Statistical Analysis}

The primary endpoint was association between FAPI-PET uptake intensity and histopathologic FAP expression. Validation via immunohistochemical FAP staining/molecular analyses of 
pathological specimen. PET uptake and tissue FAP expression will be compared by Pearson correlation for continuous data and Spearman correlation for ordinal data. In addition, uptake and expression data were compared descriptively for each score/uptake/expression range. PPV/SE on a per-patient and per-region-basis of FAPI-PET for detection of tumor location confirmed by histopathology/biopsy are calculated and reported along with the corresponding two-sided $95 \%$ confidence intervals $(\mathrm{Cl})$. The confidence intervals will be constructed using the Wilson score method. Additionally, one-way ANOVA was used for analysis of histopathological FAP expression and uptake values. Uptake measurements of tumor lesions, non-tumor lesions and background were tested for statistical difference using non-parametric paired t-tests (Wilcoxon signed rank test). All statistical analysis was performed using R statistics (version 3.4.1, www.r-project.org) or GraphPad Prism (version 8.4.2; GraphPad Software, San Diego, California USA).

\section{RESULTS}

\section{Patient Characteristics}

Until November 2020 forty-seven patients with sarcomas were enrolled at the University Hospital Essen. Four Patients were imaged with FAPI04 (8.5\%), 43 patients were imaged with FAPI46 (91.5\%). All patients imaged with FAPI46 received FDG-PET/CT $(n=35 ; 81.4 \%)$ or PET/MRI $(n$ $=8 ; 18.6 \%$ ) within a month before or after FAPI-PET (mean $3.7 \pm 6.5$ days, range -30 to +7 days). In all patients no therapeutic changes occurred in the time interval between FAPI and FDG-PET scans. Table 1 shows detailed clinical characteristics of all patients. Information on included tumor entities can be found in Supplemental Table 1. No adverse events were reported. 


\section{Association between FAPI-PET Uptake Intensity and FAP Expression}

Comparison of FAPI uptake values and histopathological FAP-score $(n=29)$ are depicted in Figure 2 and Supplemental Table 2. Histopathology samples were from needle biopsies $(n=4)$, open biopsies $(n=5)$ and surgical excisions / resections $(n=20)$. Analysis of variance of maximum standardized uptake values $\left(S U V_{\max }\right)$ and histopathological FAP-score showed significant differences $(p=0.037)$. Comparison of $S U V_{\max }$ values with the established immunohistochemical scoring system showed a moderate linear relationship $\left(\mathrm{SUV}_{\max }\right.$ Spearman $\left.r=0.43 ; p<0.05\right)$ (Fig. 2A). Higher uptake values could be observed in lesions with FAP-score 3 versus lesions with no FAP-expression (FAP-score 3 mean $23.1 \pm 15.4$ versus FAP-score 0 mean $12.0 \pm 10.2$ ). Furthermore, stratification into low- and high-grade sarcomas showed similar uptake values between groups in FAPI-PET (Fig. 2B) (Spearman's $r=-0.24 ; p=0.49$ ) with no statistically significant difference or correlation. Additional information about tracer uptake values and tracerto-background ratios are shown in the supplemental material (Supplemental Tabl. 3 and 4; Supplemental Fig. 2).

\section{Detection rate and Accuracy}

In total, 29 patients $(61.7 \%)$ and 37 regions could be validated by histopathology. The positive predictive values and sensitivity on per-patient and per-regions basis are shown in Table 2 (PPV/NPV/SE/SD contingency table in Supplemental Tabl. 5). In PET-positive patients with histopathologic validation $(n=27)$, PPV was 1.00 on a per-patient and 0.97 per-region basis (secondary endpoint; $95 \% \mathrm{Cl}, 0.87-1.00$ and $95 \% \mathrm{Cl}, 0.84-1.00$ ), respectively). In cases with histopathologic validation, 27 of $28(96 \%)$ confirmed patients and 32 of $34(94 \%)$ confirmed regions were PET positive resulting in an SE of $0.96(95 \% \mathrm{Cl}, 0.82-1.00)$ on a per-patient and 0.94 $(95 \% \mathrm{Cl}, 0.80-0.99)$ on a per-region basis (Tabl. 2). One patient was PET-negative with ruled out 
malignant lesion by histopathology (Supplemental Tabl. 5), thus accuracy was assessed for 28 patients with histopathology-confirmed malignancy.

FAPI-PET detected 310 and FDG-PET 345 lesions in total. Detailed lesion detection rate perpatient and per-region are shown in Table 3 and Supplemental Table 6. On a per patient basis detection rate was 42 (97.7\%) for FAPI-PET and 41 (95.3\%) for FDG-PET. On a per region basis $92(54.8 \%)$ regions were positive in FAPI-PET and 90 (53.6\%) in FDG-PET. A case example is shown in Figure 3.

\section{Reproducibility}

On a region basis both blinded readers showed a substantial to almost perfect agreement in both FAPI-PET and FDG-PET. Fleiss Kappa is listed in Table 4 (additional reading raw data spreadsheet as Supplemental Material 1 - 4). Especially in nodal and lung regions disease readers showed higher agreement in FAPI-PET compared to FDG-PET (local nodal $\mathrm{K}=0.54 \mathrm{vs}$ 0.27 ; distant nodal $\mathrm{K}=0.91$ vs 0.37 ; lung $\mathrm{k}=0.86$ vs 0.76 ). Inter-reader agreement of no disease vs. local disease vs metastatic disease was similar for FAPI and FDG ( $\mathrm{k}=0.71$ vs 0.72 ).

\section{Change in Management}

Completed and returned pre- and post-imaging questionnaires were available for 44 patients (93.6\%) and implemented management was assessed. As depicted in Figure 4A for 28 (64\%) of patients no change in management was documented. Major changes (e.g. change in therapeutic regimen) were documented for 7 (16\%) patients, minor changes (e.g. modification of intended therapy) occurred in $6(14 \%)$ patients. For $3(6 \%)$ of the patients no implementation of the intended treatment was reported. Types of major changes are highlighted in Figure 4B and Supplemental Table 7. The most frequent major change was treatment shift towards systemic therapy in 3 (43\%) 
patients, best supportive care in 2 (29\%) patients, active surveillance in 1 (14\%), and local therapy in $1(14 \%)$ patient. Minor changes equally distributed between modified local (50\%) and modified systemic therapy $(50 \%)$.

\section{Change in Staging}

The changes in staging between FDG-PET and FAPI-PET are given in Table 5. Five patients were excluded from the analysis due to incomparable FDG-PET datasets $(n=43)$. In most of the patients the staging did not differ between FAPI and FDG-PET. Of note, 8 (18.6\%) patients, where upstaged by FAPI-PET: 6 were upstaged from locoregional on FDG-PET to metastatic in FAPIPET and 2 patients had no signs of disease in FDG-PET and FAPI-PET resulted in staging those patients as metastatic or locoregional. As shown in Supplemental Table 7 only in two upstaged patients, this resulted in a major change in management. Downstaging by FAPI-PET occurred in $3(7 \%)$ patients.

\section{DISCUSSION}

For clinical translation of FAPI PET, association of FAPI uptake with histopathologic target expression need to be established. Previously published data indicate high uptake of FAPI tracers in sarcoma $(13,21)$. We therefore aimed to assess in patients with sarcoma the association of FAPI PET uptake with FAP expression, validated by FAP immunohistochemistry. The primary endpoint was met and we showed an association between FAPI PET uptake intensity and histopathologic FAP expression. Further we established a good diagnostic performance compared to FDG-PET in this cohort of sarcoma patients and showed high sensitivity of $96 \%$ and a PPV of 1.00 for FAP positive lesions. 
To our knowledge, this is the first prospective data on FAPI-PET in cohort of sarcoma patients and there are several strengths of this study when compared with several prior retrospective and prospective trials evaluating FAPI-PET. Our study is strengthened by a prospective follow-up, lesion validation and correlation with histopathological FAP expression, head-to-head comparison to FDG-PET, implementation of blinded reads and pre- and post-imaging questionnaires to measure management changes.

Specific binding of FAPI-04 to FAP was demonstrated previously in vitro $(15,22,23)$. In addition several clinical studies report histopathological FAP-expression in tumor lesions in smaller cohorts $(24,25)$. Nonetheless they neither compared FAP-expression level to uptake values nor showed detailed analysis of FAP-immunohistochemistry and PET lesions. We demonstrate a relationship between FAP-immunohistochemistry and $S U V_{\max }$ values. However, it has to be taken in consideration that FAP-immunohistochemistry expression level is heterogeneous in tumor tissue. The spatial intratumoral heterogeneity was addressed by assessment of the entire tumor area on the slide, but we did not perform staining on different tumor sites or metastases and for the small number of biopsies a sampling bias cannot not be excluded. Notably, seven patients had positive FAPI-PET scans whereas FAP- immunohistochemistry was negative. In this study these lesions are reported as false positive, but possible explanations for this discrepancy are sampling errors or unspecific tracer binding in areas of inflammation or necrosis (26-28).

In the assessment of diagnostic accuracy, FAPI PET demonstrates high PPV and sensitivity. When compared to FDG PET, FAPI PET had slightly lower detection efficacy for local nodal findings, however higher tumor-to-background uptake and reproducibility of interpretations. FAPIPET resulted in upstaging / downstaging in eight patients, especially from locoregional to metastatic disease compared to FDG-PET, which agrees with reported upstaging from other studies with various cancers (including three sarcoma patients) and pancreatic cancer $(29,30)$. FAPI-PET resulted in changes in treatment plans for thirteen patients. Of these, seven patients had major changes after FAPI-PET, nonetheless these changes only were triggered by upstaging 
in two of these patients. All in all, the impact of FAPI-PET on management was low, when compared to findings for PSMA PET or SSTR PET in other tumor types $(31,32)$. Reasons for a lower rate include the advanced tumor stage and pre-treatment of the enrolled patients as well as a high number of high-grade soft tissue and bone sarcoma. In high-grade sarcoma staging often does not impact the concept of induction chemotherapy and local treatment.

Aside from the diagnostic potential of FAPI radiotracers in sarcoma patients the high tracer uptake in tumor lesion enables FAP targeted radioligand therapy similar to PSMA or SSTR-targeted therapies. Recently, three cases were reported with FAP-targeted radioligand therapy, whereas one case was sarcoma with ${ }^{153}$ Samarium-labeled FAPI-46 radioligand therapy (15-17).

\section{Limitations}

Bone and soft tissue sarcomas are a group of rare and heterogenous tumors, which is reflected by variety of tumor entities included in this analysis. Due to small sample size, we were not able to perform subgroup analyses in this study, despite the possibility of differences in FAP-expression and/or FAPI-tracer uptake in certain sarcoma entities. Despite similar diagnostic performance FAPI-PET - in contrast to FDG-PET - was unable to distinguish sarcoma grades $(3,33)$.

\section{CONCLUSION}

We establish in this prospective clinical study on sarcoma patients an association between target expression and FAPI-PET SUV. We further demonstrate high accuracy of FAPI-PET and, when compared to FDG-PET, similarly high detection rate and reproducibility.

FAPI-PET is a valuable diagnostic tool in patients with sarcoma. The prognostic and theranostic potential of FAPI imaging should be explored in future studies to improve disease management. 


\section{DISCLOSURE}

L. K. is consultant for BTG and AAA and received fess from Sanofi outside of the submitted work. N.H. has nothing to disclose. J.F. has received a Junior Clinician Scientist Stipend granted by the University Duisburg-Essen. W.F. is a consultant for Endocyte and BTG, and he received fees from RadioMedix, Bayer, and Parexel outside of the submitted work. R.H. is supported by Clinician Scientist Programm of the University Medicine Essen Clinician Scientist Academy (UMEA) sponsored by faculty of medicine and Deutsche Forschungsgemeinschaft (DFG). R.H. has received travel grants from Lilly, Novartis and PharmaMar as well as fees from Lilly outside of the submitted work. M.S. reports personal fees from AstraZeneca, personal fees from Boehringer Ingelheim, personal fees from Bristol-Myers Squibb, personal fees from GlaxoSmithKline, personal fees from Janssen, personal fees from MorphoSys, personal fees from Novartis, personal fees from Takeda, personal fees from Roche, personal fees from Amgen, personal fees from MSD, outside the submitted work. L.E.P. reports personal fees from Belpharma SA, Luxembourg, outside the submitted work. U.D. has nothing to disclose. A.M. has nothing to disclose. M.C. has nothing to disclose. M.N. has nothing to disclose. F.Z. has nothing to disclose. H.U.S has nothing to disclose. L.U. has nothing to disclose. S.B. reports personal fees from Deciphera, grants from Incyte, grants and personal fees from Blueprint Medicines, personal fees from Lilly, grants and personal fees from Novartis, personal fees from Daichii-Sankyo, personal fees from Plexxikon, personal fees from Exelixis, personal fees from Bayer, other from Pfizer, during the conduct of the study; personal fees from Pharmamar, personal fees from Lilly, personal fees from Roche, personal fees from GSK, outside the submitted work. No other potential conflicts of interest relevant to this article exist.

\section{ACKNOWLEDGMENTS}

None. 


\section{KEY POINTS}

QUESTION: Is there an association of FAPI tracer uptake intensity and FAP-expression in bone and soft tissue sarcomas and what is the diagnostic performance of FAPI-PET in sarcoma patients?

PERTINENT FINDINGS: We observed an association between FAPI uptake intensity and immunhistochemical FAP-expression in sarcomas and showed high accuracy of FAPI-PET in sarcoma patients.

IMPLICATIONS FOR PATIENT CARE: Diagnostic utility of FAPI-PET for patients with sarcoma and future implications prior FAP-targeted therapies. 


\section{REFERENCES}

1. Annovazzi A, Rea S, Zoccali C, et al. Diagnostic and Clinical Impact of 18F-FDG PET/CT in Staging and Restaging Soft-Tissue Sarcomas of the Extremities and Trunk: MonoInstitutional Retrospective Study of a Sarcoma Referral Center. J Clin Med. 2020;9:2549.

2. Grimer R, Judson I, Peake D, Seddon B. Guidelines for the management of soft tissue sarcomas. Sarcoma. 2010;2010.

3. Ioannidis JPA, Lau J. 18F-FDG PET for the diagnosis and grading of soft-tissue sarcoma: A meta-analysis. J Nucl Med. 2003;44:717-724.

4. Jacob M, Chang L, Pure E. Fibroblast Activation Protein in Remodeling Tissues. Curr Mol Med. 2012.

5. Kelly T, Huang Y, Simms AE, Mazur A. Fibroblast Activation Protein- $\alpha$. A Key Modulator of the Microenvironment in Multiple Pathologies. In: International Review of Cell and Molecular Biology. ; 2012.

6. Rettig WJ, Garin-Chesa P, Beresford HR, Oettgen HF, Melamed MR, Old LJ. Cell-surface glycoproteins of human sarcomas: Differential expression in normal and malignant tissues and cultured cells. Proc Natl Acad Sci U S A. 1988.

7. Niedermeyer J, Garin-Chesa P, Kriz M, et al. Expression of the fibroblast activation protein during mouse embryo development. Int J Dev Biol. 2001.

8. Liu F, Qi L, Liu B, et al. Fibroblast activation protein overexpression and clinical implications in solid tumors: A meta-analysis. PLoS One. 2015;10:1-18.

9. Koczorowska MM, Tholen S, Bucher F, et al. Fibroblast activation protein- $\alpha$, a stromal cell surface protease, shapes key features of cancer associated fibroblasts through proteome 
and degradome alterations. Mol Oncol. 2016.

10. Kalluri R. The biology and function of fibroblasts in cancer. Nat Rev Cancer. 2016;16:582598.

11. Dohi $\mathrm{O}$, Ohtani $\mathrm{H}$, Hatori $\mathrm{M}$, et al. Histogenesis-specific expression of fibroblast activation protein and dipeptidylpeptidase-IV in human bone and soft tissue tumours. Histopathology. 2009;55:432-440.

12. Scanlan MJ, Raj BKM, Calvo B, et al. Molecular cloning of fibroblast activation protein $\alpha$, a member of the serine protease family selectively expressed in stromal fibroblasts of epithelial cancers. Proc Natl Acad Sci U S A. 1994;91:5657-5661.

13. Kratochwil C, Flechsig P, Lindner T, et al. FAPI-PET/CT: Mean intensity of tracer-uptake (SUV) in 28 different kinds of cancer. J Nucl Med. 2019:jnumed.119.227967.

14. Giesel FL, Kratochwil C, Lindner T, et al. 68 Ga-FAPI PET/CT: Biodistribution and Preliminary Dosimetry Estimate of 2 DOTA-Containing FAP-Targeting Agents in Patients with Various Cancers. J Nucl Med. 2019;60:386-392.

15. Lindner T, Loktev A, Altmann A, et al. Development of Quinoline-Based Theranostic Ligands for the Targeting of Fibroblast Activation Protein. J Nucl Med. 2018;59:14151422.

16. Ballal S, Yadav MP, Kramer V, et al. A theranostic approach of [68Ga]Ga-DOTA.SA.FAPi PET/CT-guided [177Lu]Lu-DOTA.SA.FAPi radionuclide therapy in an end-stage breast cancer patient: new frontier in targeted radionuclide therapy. Eur J Nucl Med Mol Imaging. 2020:1-3.

17. Kratochwil C, Giesel FL, Rathke H, et al. [153Sm]Samarium-labeled FAPI-46 radioligand therapy in a patient with lung metastases of a sarcoma. Eur J Nucl Med Mol Imaging. March 2021:8-10. 
18. Henry LR, Lee HO, Lee JS, et al. Clinical implications of fibroblast activation protein in patients with colon cancer. Clin Cancer Res. 2007;13:1736-1741.

19. Iwasa S, Jin X, Okada K, Mitsumata M, Ooi A. Increased expression of seprase, a membrane-type serine protease, is associated with lymph node metastasis in human colorectal cancer. Cancer Lett. 2003.

20. Ariga N, Sato E, Ohuchi N, Nagura H, Ohtani H. Stromal expression of fibroblast activation protein/seprase, a cell membrane serine proteinase and gelatinase, is associated with longer survival in patients with invasive ductal carcinoma of breast. Int $J$ Cancer. 2001;95:67-72.

21. Giesel F, Kratochwil C, Lindner T, et al. FAPI-PET/CT: biodistribution and preliminary dosimetry estimate of two DOTA-containing FAP-targeting agents in patients with various cancers. J Nucl Med. August 2018:jnumed.118.215913.

22. Loktev A, Lindner T, Mier W, et al. A new method for tumor imaging by targeting cancer associated fibroblasts. J Nucl Med. 2018:jnumed.118.210435.

23. Loktev A, Lindner T, Burger E-M, et al. Development of Fibroblast Activation ProteinTargeted Radiotracers with Improved Tumor Retention. J Nucl Med. 2019;60:1421-1429.

24. Röhrich M, Loktev A, Wefers AK, et al. IDH-wildtype glioblastomas and grade III/IV IDHmutant gliomas show elevated tracer uptake in fibroblast activation protein-specific PET/CT. Eur J Nucl Med Mol Imaging. 2019;46:2569-2580.

25. Shi X, Xing H, Yang X, et al. Comparison of PET imaging of activated fibroblasts and 18FFDG for diagnosis of primary hepatic tumours: a prospective pilot study. Eur J Nucl Med Mol Imaging. October 2020.

26. Chen H, Zhao L, Ruan D, et al. Usefulness of [(68)Ga]Ga-DOTA-FAPI-04 PET/CT in patients presenting with inconclusive [(18)F]FDG PET/CT findings. Eur J Nucl Med Mol 
Imaging. June 2020.

27. Luo Y, Pan Q, Zhang W, Li F. Intense FAPI Uptake in Inflammation May Mask the Tumor Activity of Pancreatic Cancer in 68Ga-FAPI PET/CT. Clin Nucl Med. 2020;45:310-311.

28. Schmidkonz C, Rauber S, Atzinger A, et al. Disentangling inflammatory from fibrotic disease activity by fibroblast activation protein imaging. Ann Rheum Dis. 2020;79:14851491.

29. Chen H, Pang Y, Wu J, et al. Comparison of [68Ga]Ga-DOTA-FAPI-04 and [18F] FDG $\mathrm{PET} / \mathrm{CT}$ for the diagnosis of primary and metastatic lesions in patients with various types of cancer. Eur J Nucl Med Mol Imaging. 2020;47:1820-1832.

30. Röhrich M, Naumann P, Giesel FL, et al. Impact of 68 Ga-FAPI-PET/CT imaging on the therapeutic management of primary and recurrent pancreatic ductal adenocarcinomas . $J$ Nucl Med. 2020:jnumed.120.253062.

31. Calais J, Czernin J, Eiber M, et al. Most of the Intended Management Changes After 68 Ga-DOTATATE PET/CT Are Implemented. J Nucl Med. 2017;58:1793-1796.

32. Fendler WP, Ferdinandus J, Czernin J, et al. Impact of 68Ga-PSMA-11 PET on the Management of Recurrent Prostate Cancer in a Prospective Single-Arm Clinical Trial. J Nucl Med. 2020;61:1793-1799.

33. Eary JF, Conrad EU, Bruckner JD, et al. Quantitative [F-18]fluorodeoxyglucose positron emission tomography in pretreatment and grading of sarcoma. Clin Cancer Res. 1998;4:1215-1220. 


\section{Tables}

Table 1. Patient characteristics. Data are n (\%).

\begin{tabular}{|c|c|}
\hline & $\begin{array}{l}\text { Overall } \\
(n=47)\end{array}$ \\
\hline \multicolumn{2}{|l|}{ Age } \\
\hline Mean (SD) & $48.1(17.3)$ \\
\hline Median [Min, Max] & $54.0[18.0,89.0]$ \\
\hline \multicolumn{2}{|l|}{ Gender } \\
\hline female & $23(48.9 \%)$ \\
\hline male & $24(51.1 \%)$ \\
\hline \multicolumn{2}{|l|}{ Sarcoma type } \\
\hline Bone sarcoma & $20(42.6 \%)$ \\
\hline Osteosarcoma & $8(17.0 \%)$ \\
\hline Ewing-sarcoma & $2(4.3 \%)$ \\
\hline others & $10(21.3 \%)$ \\
\hline Soft tissue sarcoma & $27(57.4 \%)$ \\
\hline NOS / UPS & $6(12.8 \%)$ \\
\hline Leiomyosarcoma & $4(8.5 \%)$ \\
\hline others & $17(36.2 \%)$ \\
\hline \multicolumn{2}{|l|}{ Grading } \\
\hline high-grade & $35(74.5 \%)$ \\
\hline low grade & $5(10.6 \%)$ \\
\hline not applicable & $7(14.9 \%)$ \\
\hline \multicolumn{2}{|l|}{ Staging } \\
\hline Localized & $16(34.0 \%)$ \\
\hline
\end{tabular}




\begin{tabular}{|c|c|}
\hline & $\begin{array}{l}\text { Overall } \\
(n=47)\end{array}$ \\
\hline Synchrone metastasized & $9(19.1 \%)$ \\
\hline Metachrone metastasized & $22(46.8 \%)$ \\
\hline \multicolumn{2}{|l|}{ Prior therapies } \\
\hline Chemotherapy & $13(27.7 \%)$ \\
\hline Chemotherapy and Radiotherapy & $15(31.9 \%)$ \\
\hline Radiotherapy & $2(4.3 \%)$ \\
\hline None & $17(36.2 \%)$ \\
\hline \multicolumn{2}{|l|}{ Indication for PET } \\
\hline Staging & $17(36.2 \%)$ \\
\hline Re-Staging & $20(42.6 \%)$ \\
\hline Localization before local therapy & $10(21.3 \%)$ \\
\hline \multicolumn{2}{|l|}{ Intended treatment before PET } \\
\hline Chemotherapy & $26(55.3 \%)$ \\
\hline Resection & $12(25.5 \%)$ \\
\hline Radiation & $5(10.6 \%)$ \\
\hline Resection / Radiation & $2(4.3 \%)$ \\
\hline Watch \& wait & $2(4.3 \%)$ \\
\hline
\end{tabular}


Table. 2. FAPI-PET Accuracy. Positive predictive value and sensitivity of FAPI-PET confirmed by histopathological validation on a per-patient and per-region basis.

\begin{tabular}{lllll}
\hline & Total Regions/ & No. (\%) & \\
Validation group & Patients, No. & Confirmed & Ruled out & PPV or SE (95\% Cl) \\
\hline Positive predictive value & & & \\
\hline Histopathologic validation & & $27(100)$ & $0(0)$ & 1.00 (0.87 to 1.00) \\
\hline PET-positive (per-patient) & 27 & $32(97)$ & $1(3)$ & $0.97(0.84$ to 1.00) \\
\hline PET-positive (per-region) & 33 & & & \\
\hline Sensitivity & & $27(96)^{\mathrm{a}}$ & $1(4)^{\mathrm{b}}$ & 0.96 (0.82 to 1.00) \\
\hline Histopathologic findings & & $32(94)^{\mathrm{a}}$ & $2(6)^{\mathrm{b}}$ & 0.94 (0.80 to 0.99) \\
\hline confirmed (per-patient) & 28 & 34 & & \\
\hline confirmed (per-region) & & & & \\
\hline
\end{tabular}

Abbreviations: PET, positron emission tomography; PPV, positive predictive value; SE, sensitivity. aPET positive. ${ }^{\mathrm{b} P E T}$ negative. 
Table 3. Detection efficacy.

\begin{tabular}{|c|c|c|}
\hline & \multicolumn{2}{|c|}{ PET-positive results, No. (\%) } \\
\hline & FAPI $(n=43)$ & FDG $(n=43)$ \\
\hline Per-patient basis & $42(97.7 \%)$ & $41(95.3 \%)$ \\
\hline \multicolumn{3}{|l|}{ Per-region basis } \\
\hline Primary & $33(76.7 \%)$ & $35(81.4 \%)$ \\
\hline Local nodal & $5(11.6 \%)$ & $7(16.3 \%)$ \\
\hline Distant nodal & $5(11.6 \%)$ & $8(18.6 \%)$ \\
\hline Lung & $15(34.9 \%)$ & $14(32.6 \%)$ \\
\hline Bone & $12(27.9 \%)$ & $9(20.9 \%)$ \\
\hline Other & $23(53.5 \%)$ & $16(37.2 \%)$ \\
\hline
\end{tabular}

Abbreviations: PET, positron emission tomography; FAPI, fibroblast-activation protein inhibitor; FDG, fluorodeoxyglucose 
Table 4. Reproducibility. Interreader agreement (Fleiss' Kappa) is shown on a per-region basis for FDG-PET and FAPI-PET.

\begin{tabular}{ccc}
\hline & \multicolumn{3}{c}{ Fleiss' $\mathbf{~}$} \\
\cline { 2 - 3 } & FDG $(\mathrm{n}=43)$ & FAPI $(\mathrm{n}=47)$ \\
\hline per-region & 0.77 & 0.78 \\
\hline primary & 0.27 & 0.54 \\
\hline local nodal & 0.37 & 0.91 \\
\hline distant nodal & 0.78 & 0.86 \\
\hline lung & 0.66 & 0.69 \\
\hline bone & 0.67 & 0.65 \\
\hline other & & 0.706 \\
\hline No disease vs. & 0.718 & \\
\hline Local disease vs & & \\
\hline Metastatic disease & & \\
\hline
\end{tabular}


Table 5. Change in stage by addition of FAPI PET.

\begin{tabular}{lccc}
\hline FDG & $\begin{array}{c}\text { locoregional } \\
(\mathbf{n}=13)\end{array}$ & $\begin{array}{c}\text { metastatic } \\
(\mathbf{n}=\mathbf{2 9})\end{array}$ & $\begin{array}{c}\text { No disease } \\
(\mathbf{n}=\mathbf{1})\end{array}$ \\
\hline locoregional & $10(76.9 \%)$ & $6(20.7 \%)$ & $1(100 \%)$ \\
metastatic & $2(15.4 \%)$ & $22(75.9 \%)$ & $0(0 \%)$ \\
No Disease & $1(7.7 \%)$ & $1(3.4 \%)$ & $0(0 \%)$ \\
\hline
\end{tabular}




\section{Figures}

\section{Figure 1. Enrollment Flowchart}

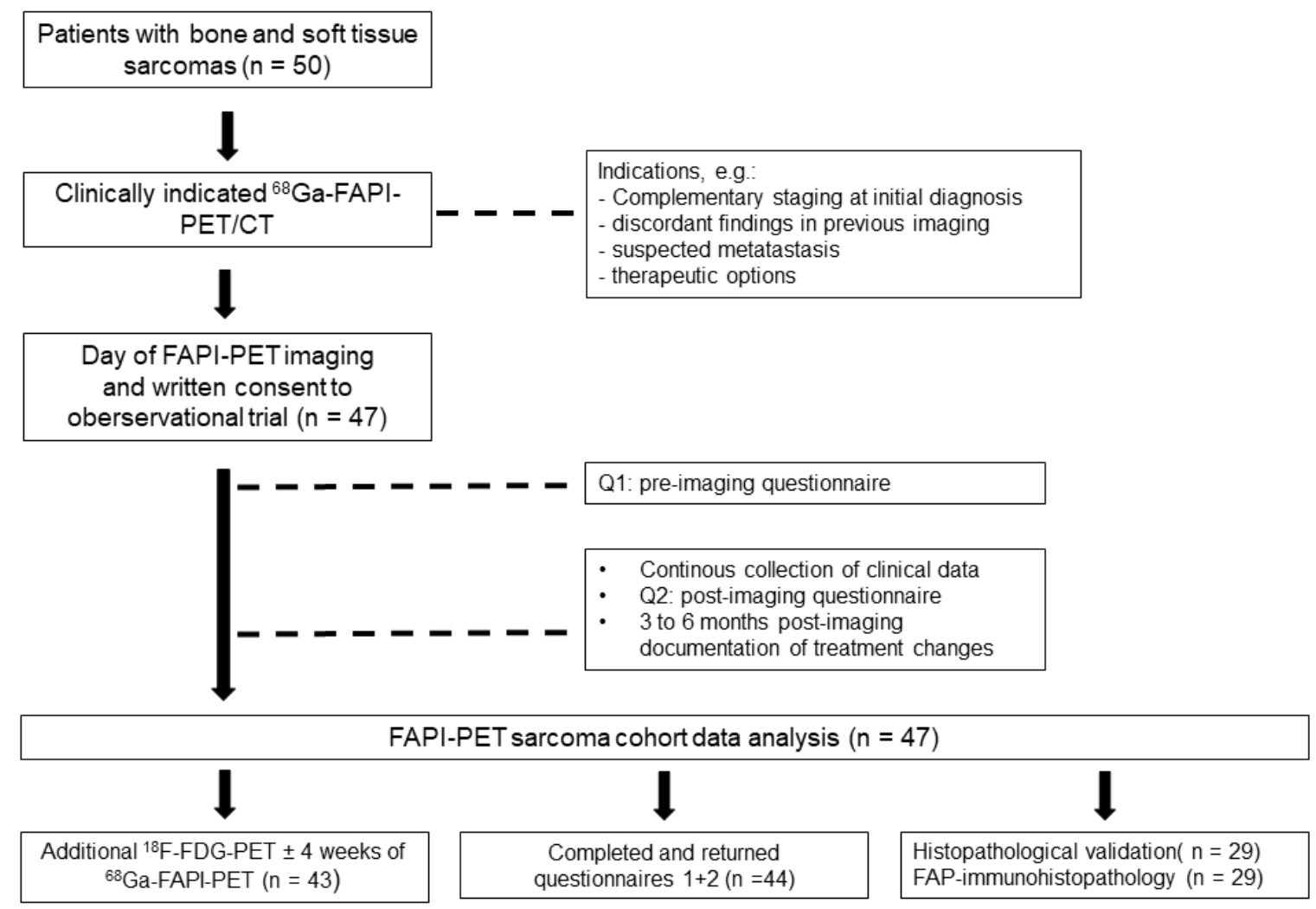


Figure 2. Primary Endpoint. Association between FAPI-PET uptake intensity and FAP IHC score. (A) shows ${ }^{68} \mathrm{Ga}-\mathrm{FAPI} \mathrm{SUV}_{\max }$ by IHC-Score with positive correlation $(r=0.43, p<0.05)$. (B) shows ${ }^{68} \mathrm{Ga}-F A P I ~ S U V_{\max }$ for patients separated into high grade and low-grade groups and patients with tumor entities where grading does not apply. Uptake values do not show statistically significant difference between groups $(p=0.49)$.

A

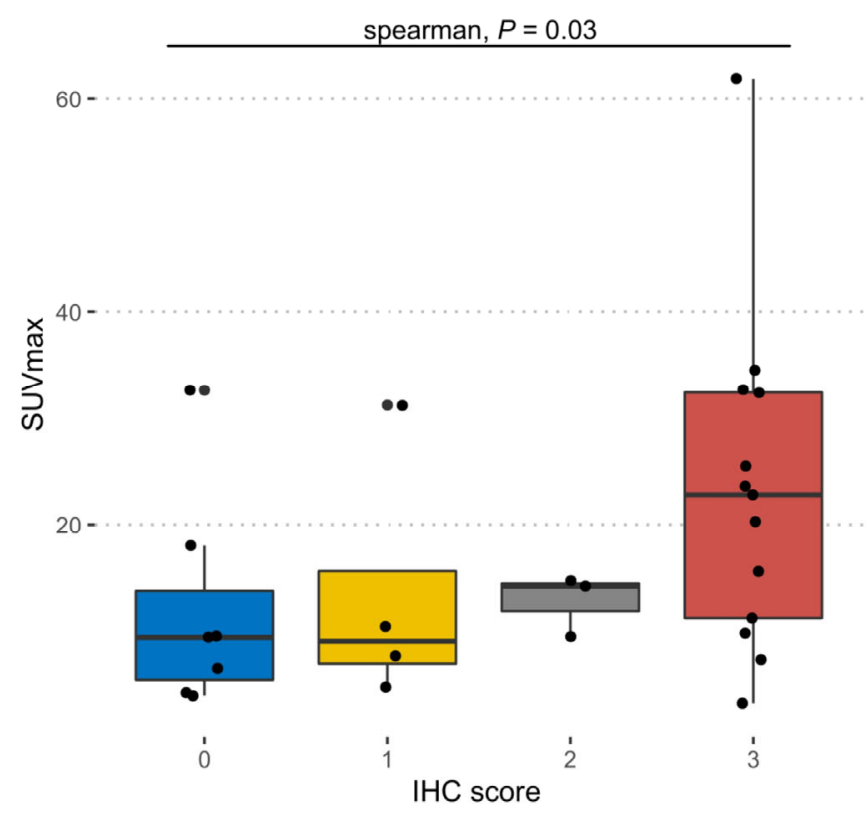

B

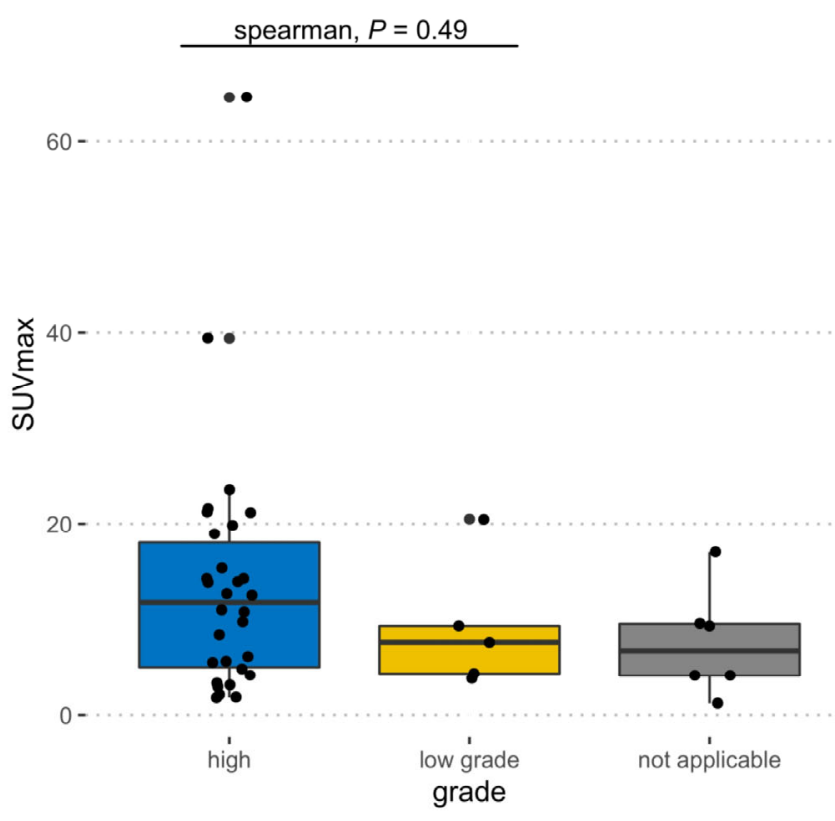


Figure 3. Case presentation. 69-year-old patient with metastatic low-grade myofibroblastic sarcoma. Maximum-intensity projection of FAPI-PET $(\mathbf{A})$ and axial CT and PET imaging (B) of the primary tumor lesion of the dorsal left thigh show high uptake values in FAPI-PET (SUV $V_{\max }$ 34.5) vs. FDG-PET axial imaging (SUV $\max 20.6)$ (C) and maximum-intensity projections (D) . The primary lesion (blue arrow) demonstrated high FAP expression in IHC (E) compared to negative immunohistochemistry of a different patient $(\mathbf{F})$.
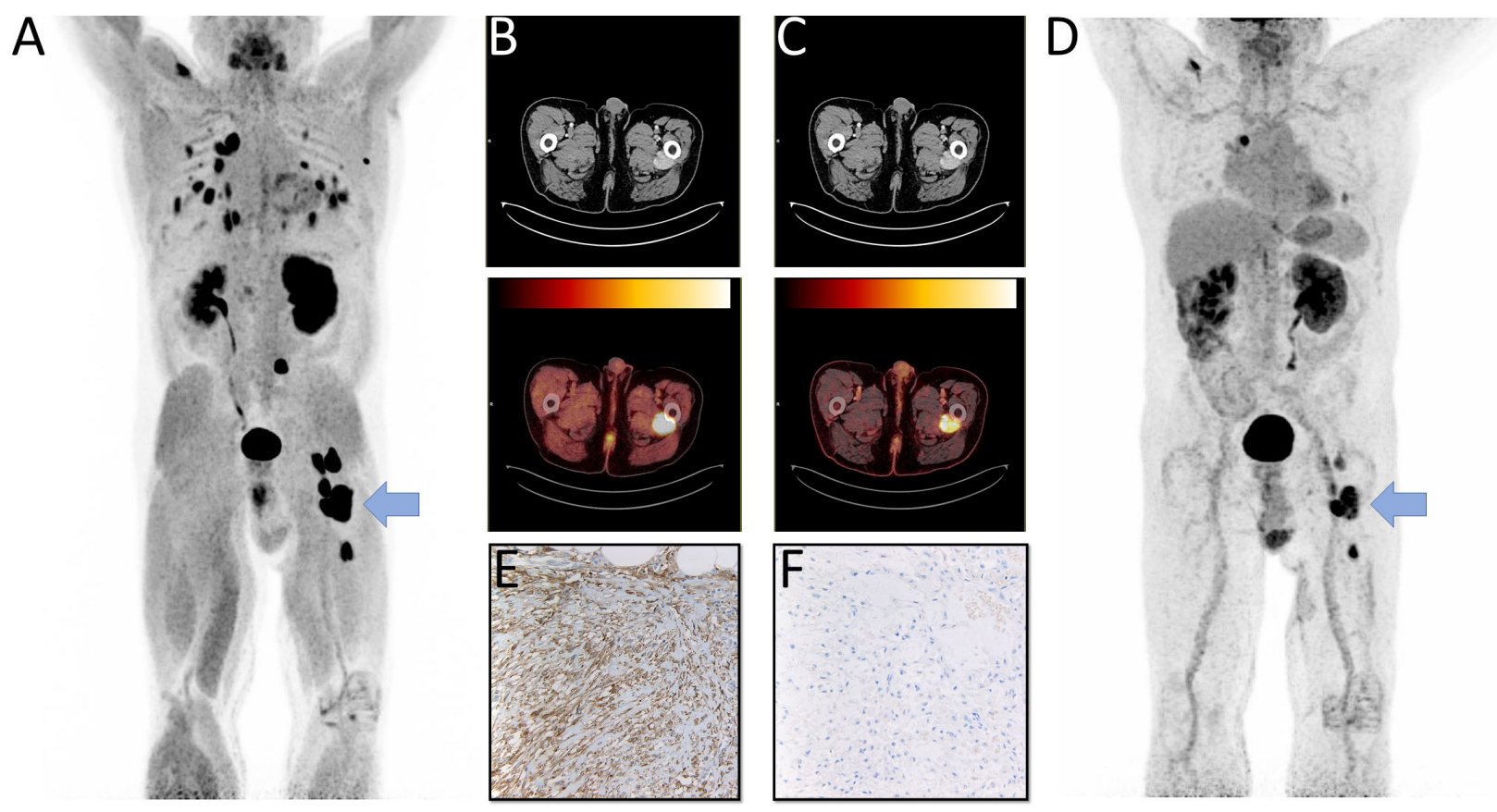
Figure 4. Change in management. (A) pie chart of implemented management change after FAPI-PET for 44 patients. Types of changes were categorized as no change, minor change (e.g. modification of systemic or local treatment), major change (e.g. treatment shift towards local or systemic therapy) and no report of implementation of intended treatment. (B) shows the types of major change after FAPI-PET in 7 patients. Major shift towards systemic therapy were noted most often.

A

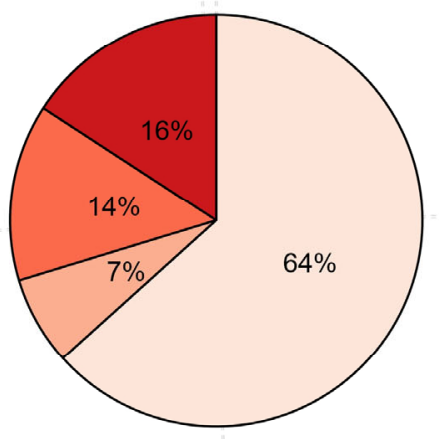

B

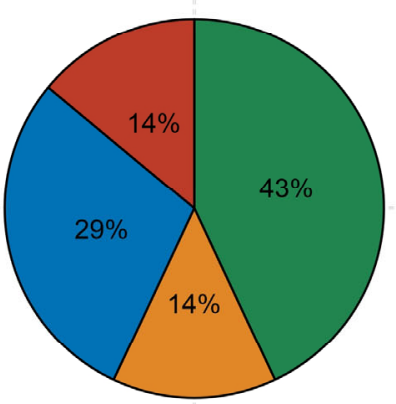

Type of major change

Toward active surveillance Towards best supportive care Towards local therapy

Towards systemic therapy 


\section{Supplement}

\section{Supplemental Table 1. Histology detail}

Overall

$(n=47)$

\begin{tabular}{|c|c|}
\hline \multicolumn{2}{|l|}{ Histology } \\
\hline Angiosarcoma & $1(2.0 \%)$ \\
\hline Chordoma & $6(12.0 \%)$ \\
\hline Dedifferentiated chondrosarcoma & $1(2.0 \%)$ \\
\hline Endometrial stromal sarcoma & $2(4.0 \%)$ \\
\hline Ewing Sarcoma & $2(4.0 \%)$ \\
\hline Gastrointestinal stromal tumor & $2(4.0 \%)$ \\
\hline Leiomyosarcoma & $4(8.0 \%)$ \\
\hline Low-grade myofibroblastic sarcoma & $1(2.0 \%)$ \\
\hline Malignant solitary fibrous tumor & $2(4.0 \%)$ \\
\hline Myxoid liposarcoma & $3(6.0 \%)$ \\
\hline Malignant peripheral nerve sheath tumor & $1(2.0 \%)$ \\
\hline Mixed Müllerian tumor & $1(2.0 \%)$ \\
\hline Myxofibrosarcoma & $2(4.0 \%)$ \\
\hline Undifferentiated soft tissue sarcoma (NOS, round cell, UPS) & $6(12.0 \%)$ \\
\hline Undifferentiated bone sarcoma & $2(4.0 \%)$ \\
\hline Osteosarcoma & $8(16.0 \%)$ \\
\hline Rhabdomyosarcoma of the bone & $1(2.0 \%)$ \\
\hline Synovialsarcoma & $2(4.0 \%)$ \\
\hline
\end{tabular}


Supplemental Table 2. FAPI uptake vs IHC on a per patient basis. IHC scoring: $0=$ no expression (<1\%), 1+=1-10\%, 2+=11-49\%, 3+= $\geq 50 \%$ FAP-positive cells.

\begin{tabular}{|c|c|c|c|c|c|}
\hline & $\begin{array}{l}\text { IHC } 0 \\
(n=8)\end{array}$ & $\begin{array}{l}\text { IHC } 1 \\
(n=4)\end{array}$ & $\begin{array}{l}\text { IHC } 2 \\
(n=3)\end{array}$ & $\begin{array}{l}\text { IHC 3 } \\
(n=13)\end{array}$ & $\begin{array}{l}\text { Overall } \\
(n=47)\end{array}$ \\
\hline \multicolumn{6}{|l|}{ SUV $\max$ tumor } \\
\hline Mean (SD) & $12.0(10.2)$ & $13.5(12.0)$ & $12.8(2.87)$ & $23.1(15.4)$ & $15.2(11.7)$ \\
\hline $\begin{array}{l}\text { Median [Min, } \\
\text { Max] }\end{array}$ & $\begin{array}{c}9.40[3.90 \\
32.6]\end{array}$ & $\begin{array}{c}9.05[4.70 \\
31.2]\end{array}$ & $\begin{array}{c}14.2[9.50 \\
14.7]\end{array}$ & $\begin{array}{c}22.8[3.20 \\
61.9]\end{array}$ & $\begin{array}{c}10.8[3.20, \\
61.9]\end{array}$ \\
\hline
\end{tabular}


Supplemental Table 3. FAPI and FDG uptake for high versus low grade tumors. In 7 patients tumor grading was not applicable.

\begin{tabular}{lcccc}
\hline & $\begin{array}{c}\text { high grade } \\
(\mathbf{n}=\mathbf{3 5})\end{array}$ & $\begin{array}{c}\text { low grade } \\
(\mathbf{n}=5)\end{array}$ & $\begin{array}{c}\text { not applicable } \\
(\mathbf{n = 7})\end{array}$ & $\begin{array}{c}\text { Overall } \\
(\mathbf{n}=\mathbf{4 7})\end{array}$ \\
\hline FAPI & & & & \\
\hline Mean (SD) & $14.2(12.0)$ & $21.1(12.5)$ & $15.8(9.52)$ & $15.2(11.7)$ \\
Median [Min, Max] & $9.50[3.60,61.9]$ & $18.0[4.70,34.5]$ & $12.4[3.20,27.8]$ & $10.8[3.20,61.9]$ \\
\hline FDG & & & & \\
\hline Mean (SD) & $13.6(12.8)$ & $9.10(6.77)$ & $7.55(5.67)$ & $12.2(11.6)$ \\
\hline Median [Min, Max] & $11.8[1.80,64.6]$ & $7.60[3.80,20.5]$ & $6.70[1.20,17.0]$ & $9.60[1.20,64.6]$ \\
\hline
\end{tabular}




\section{Supplemental Table 4. Tracer-to-Background}

\begin{tabular}{|c|c|c|}
\hline & $\begin{array}{c}\text { FAPI } \\
(n=47)\end{array}$ & $\begin{array}{c}\text { FDG } \\
(n=43)\end{array}$ \\
\hline \multicolumn{3}{|l|}{ SUV $\max$ tumor } \\
\hline Mean (SD) & $15.2(11.7)$ & $12.2(11.6)$ \\
\hline Median [Min, Max] & $10.8[3.20,61.9]$ & $9.60[1.20,64.6]$ \\
\hline \multicolumn{3}{|l|}{ TBR liver } \\
\hline Mean (SD) & $18.0(14.8)$ & $7.20(8.22)$ \\
\hline Median [Min, Max] & $14.1[2.83,78.0]$ & $4.65[0.800,43.1]$ \\
\hline \multicolumn{3}{|l|}{ TBR blood pool } \\
\hline Mean (SD) & $10.5(8.39)$ & $8.28(8.10)$ \\
\hline Median [Min, Max] & $7.47[1.89,34.4]$ & $5.68[0.947,38.0]$ \\
\hline \multicolumn{3}{|l|}{ TBR muscle } \\
\hline Mean (SD) & $11.8(9.76)$ & $24.3(29.3)$ \\
\hline Median [Min, Max] & $7.50[2.46,41.3]$ & 16.0 [2.0 to 162.0$]$ \\
\hline
\end{tabular}


Supplemental Table 5. SE, SD, PPV and NPV on a per patient basis for histopathologically confirmed lesions.

\begin{tabular}{cccccc}
\hline & $\begin{array}{c}\text { Histopathol. } \\
\text { confirmed }\end{array}$ & $\begin{array}{c}\text { Histopathol. } \\
\text { ruled out }\end{array}$ & Total & & \\
\hline PET positive & $\mathbf{2 7}$ & $\mathbf{0}$ & $\mathbf{2 7}$ & PPV & $100 \%$ \\
\hline PET negative & $\mathbf{1}$ & $\mathbf{1}$ & $\mathbf{2}$ & NPV & $50 \%$ \\
\hline Total & $\mathbf{2 8}$ & $\mathbf{1}$ & $\mathbf{2 9}$ & & \\
\hline & Sensivity & Specificity & & \\
& $96 \%$ & $100 \%$ & &
\end{tabular}


Supplemental Table 6. Lesion number on a per-patient and per-region basis.

\begin{tabular}{|c|c|c|}
\hline & \multicolumn{2}{|c|}{ PET-positive lesions, No. } \\
\hline & FAPI $(n=43)$ & FDG $(n=43)$ \\
\hline Per-patient basis & 310 & 345 \\
\hline \multicolumn{3}{|l|}{ Per-region basis } \\
\hline Primary & 46 & 48 \\
\hline Local nodal & 10 & 13 \\
\hline Distant nodal & 19 & 35 \\
\hline Lung & 94 & 110 \\
\hline Bone & 56 & 40 \\
\hline Other & 85 & 99 \\
\hline
\end{tabular}

Abbreviations: PET, positron emission tomography; FAPI, fibroblast-activation protein inhibitor; FDG, fluorodeoxyglucose 
Supplemental Table 7. List of patients with major change in treatment.

\begin{tabular}{|c|c|c|c|c|}
\hline $\begin{array}{c}\text { Patient } \\
\text { no. }\end{array}$ & Intended treatment & Managment towards & Change in stage by FAPI-PET & $\begin{array}{c}\text { Disease } \\
\text { extent }\end{array}$ \\
\hline \multirow{2}{*}{1} & \multirow{2}{*}{ Radio-/Chemotherapy } & \multirow{2}{*}{ Best supportive care } & Upstaged & \multirow{2}{*}{ increase } \\
\hline & & & local to metastastic & \\
\hline \multirow{2}{*}{2} & \multirow{2}{*}{$\begin{array}{l}\text { Local } \\
\text { radiochemotherapy }\end{array}$} & \multirow{2}{*}{ Systemic therapy } & No change & \multirow{2}{*}{ decrease } \\
\hline & & & Metastatic to metastatic & \\
\hline \multirow{2}{*}{3} & \multirow{2}{*}{$\begin{array}{l}\text { Local } \\
\text { radiochemotherapy }\end{array}$} & \multirow{2}{*}{ Systemic therapy } & Upstaged & \multirow{2}{*}{ increase } \\
\hline & & & No disease to local disease & \\
\hline \multirow[b]{2}{*}{4} & \multirow{2}{*}{$\begin{array}{c}\text { Neoadjuvant } \\
\text { chemotherapy and } \\
\text { surgery }\end{array}$} & \multirow[b]{2}{*}{ Systemic therapy } & Downstaged & \multirow[b]{2}{*}{ decrease } \\
\hline & & & Local to no disease & \\
\hline \multirow{2}{*}{5} & \multirow{2}{*}{ Radio-/Chemotherapy } & \multirow{2}{*}{ Active surveillance } & No change & \multirow{2}{*}{ equal } \\
\hline & & & Local to local & \\
\hline \multirow{2}{*}{6} & \multirow{2}{*}{ Chemotherapy } & \multirow{2}{*}{ Local therapy (radiation therapy) } & No change & \multirow{2}{*}{ increase } \\
\hline & & & Metastatic to metastatic & \\
\hline \multirow{2}{*}{7} & \multirow{2}{*}{$\begin{array}{c}\text { Chemotherapy and } \\
\text { surgery }\end{array}$} & \multirow{2}{*}{ Best supportive care } & No change & \multirow{2}{*}{ equal } \\
\hline & & & Metastatic to metastatic & \\
\hline
\end{tabular}




\section{Supplemental Figures}

Supplemental Figure 1. Timeline of investigations. Study timelines are shown in blue.

Procedures as per clinical routine are shown in green.

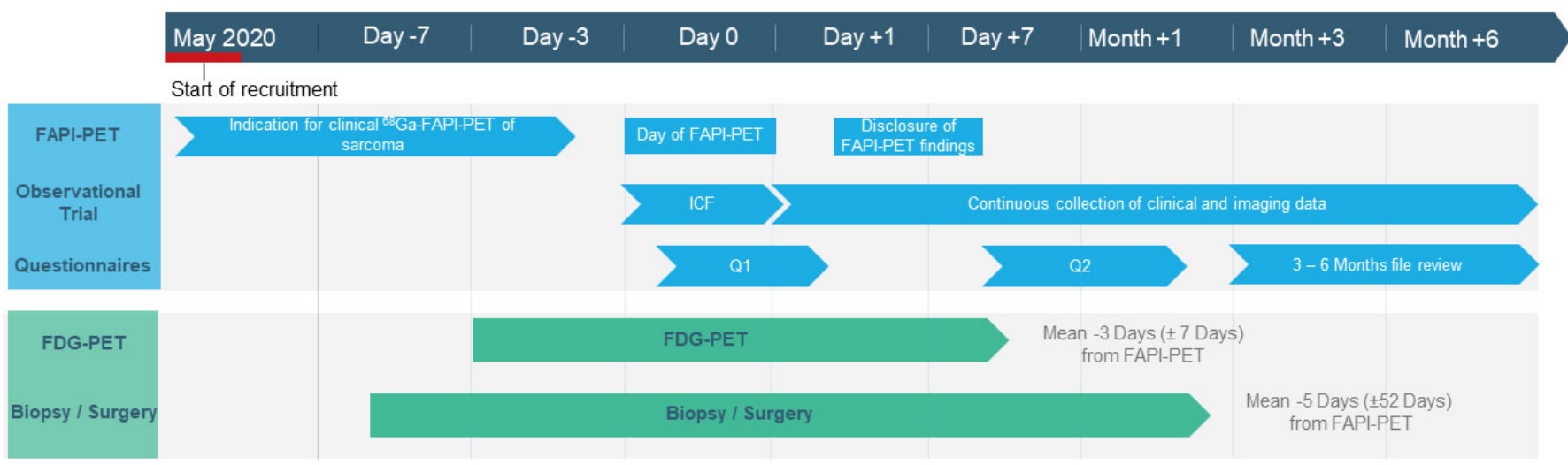




\section{Supplemental Figure 2. Mean and maximum organ uptake and tumor to background}

ratios (TBRs). Left diagramm shows SUV max for FAPI and FDG in tumor lesions in the predefined regions. Further SUV mean for background tracer uptake shows lower background

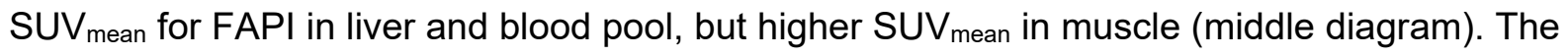
right diagram shows higher TBRs for FAPI vs FDG normalized by liver uptake but lower TBR by normalization to muscle uptake.

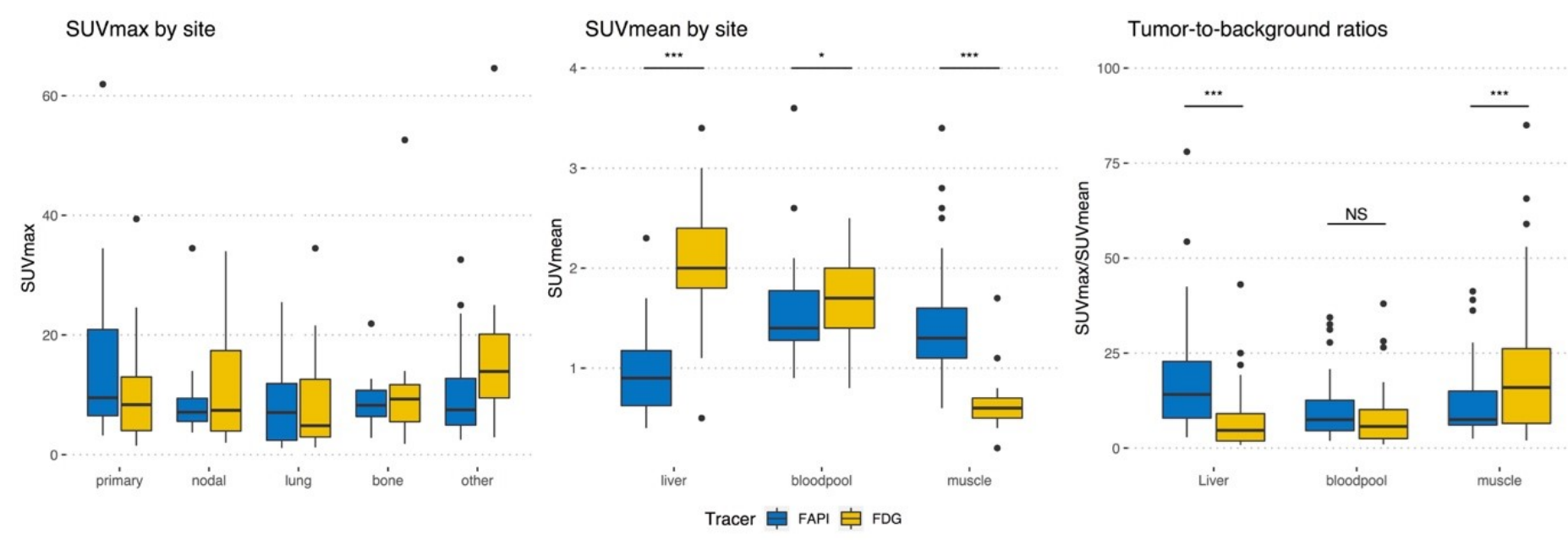


Supplemental Material

Supplemental Material 1. Reading Raw Data. Excel-spreadsheet of reading raw data for Reader 1 FAPI-PET interpretation.

Supplemental Material 2. Reading Raw Data. Excel-spreadsheet of reading raw data for Reader 1 FDG-PET interpretation.

Supplemental Material 3. Reading Raw Data. Excel-spreadsheet of reading raw data for Reader 2 FAPI-PET interpretation.

Supplemental Material 4. Reading Raw Data. Excel-spreadsheet of reading raw data for Reader 2 FDG-PET interpretation. 


\section{GRAPHICAL ABSTRACT}

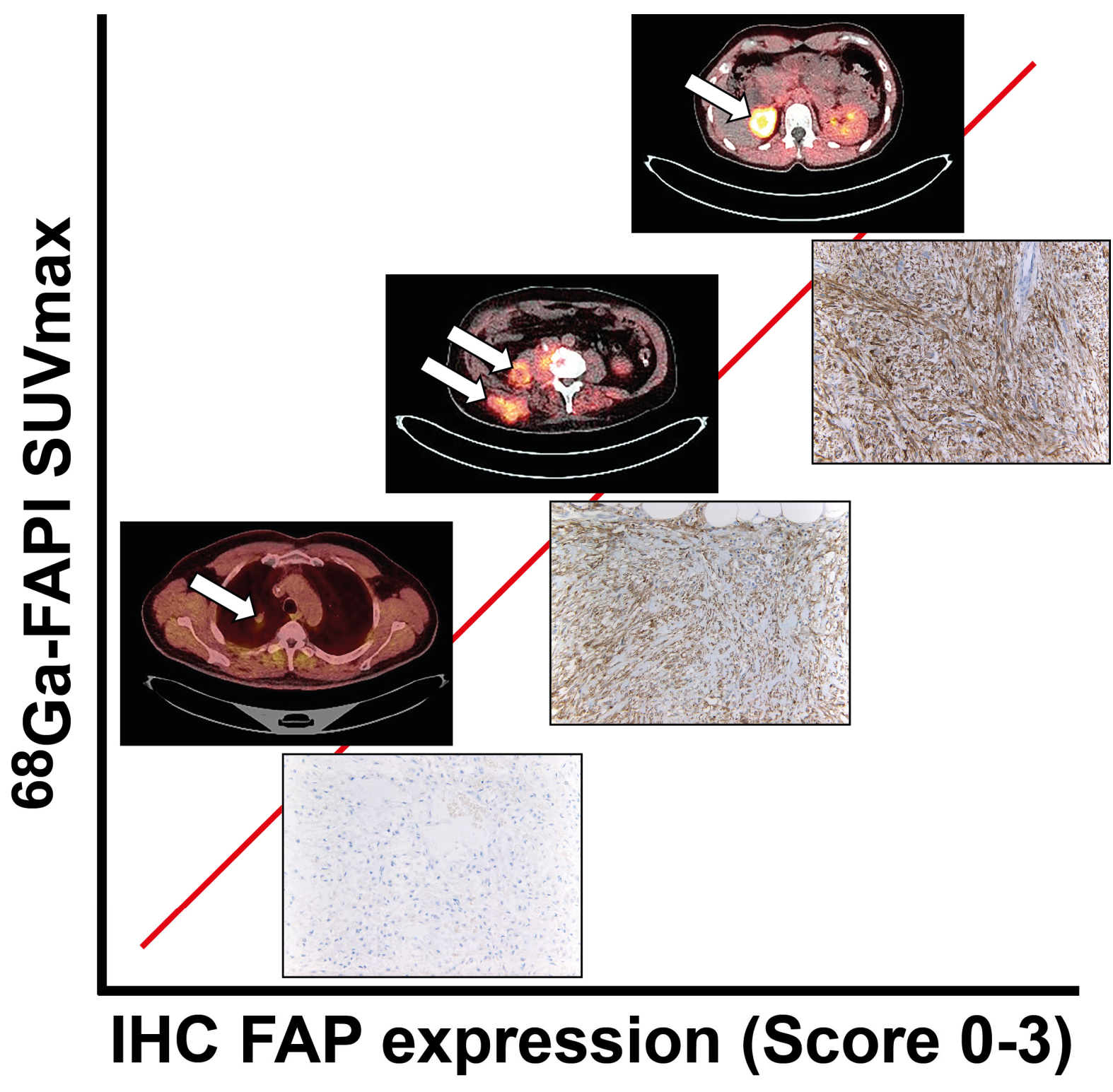

